\title{
Clinical Characteristics and Degree of Severity of COVID-19 in Hemodialysis Patients in Armed Forces Institute Of Urology
}

\author{
Brig. Sohail Sabir, Saba Umar, Brig. Khalid Raja ${ }^{2}$, Lt.col Khurram Mansoor ${ }^{1}$, Lt. \\ col. Tanveer Sajid ${ }^{1}$, Maj. Gen Salman Saleem², Maj Gen Aamir Ikram ${ }^{3}$, Brig. Fuad \\ Ahmed Siddqui ${ }^{4}$, Surg Cdre. Haroon Sabir ${ }^{5}$
}

\author{
${ }^{1}$ Armed Forces Institute of Urology Rawalpindi, Pakistan. \\ 2Pak Emirates Military Hospital Rawalpindi, Pakistan \\ ${ }^{3}$ National Institute of Health, Islamabad, Pakistan \\ ${ }^{4} \mathrm{CMH}$ Rawalpindi, Pakistan \\ ${ }^{5}$ PNS shifa, Karachi, Pakistan
}

\begin{abstract}
Introduction

This study was conducted to evaluate the clinical characteristics and severity of COVID -19 in hemodialysis patients at Armed Forces Hospital of Urology, Rawalpindi Pakistan from $1^{\text {st }}$ March 2020 to $15^{\text {th }}$ August 2020.

Methodology

It was a prospective and Cross Sectional Observational Study. We collected data prospectively that includes all patients on maintenance hemodialysis and reviewed clinical characteristics of those with laboratory-confirmed COVID-19 between March 1and August 15, 2020.

Results

39 out of 268 dialysis dependent patients had COVID-19. Mean age of patients was 55.9yrs. Only 35.8\% patients were symptomatic. 15 out of 39 were having mild disease, 12 had moderate and 12 had severe disease. Females (54.5\%) were found to be more affected than males (45.5\%). Dry cough was the commonest symptom (53.8\%) followed by fever $(46.1 \%)$ and abdominal pain(18.1\%). Patients with multiple comorbidities were found to have severe disease.

Conclusion

We concluded that patients receiving maintenance hemodialysis are susceptible to COVID-19 and that hemodialysis centers are at high risk for spread of infection. Isolating patients with COVID-19 can help in preventing the spread of COVID-19.
\end{abstract}

Key words: covid-19, hemodialysis, pandemic, comorbidity, diabetes mellitus, ischemic heart disease.

\section{Corresponding author}

Brig. Sohail Sabir,

Head of Nephrology

Armed Forces Institute of Urology Rawalpindi, Pakistan.

Email: drsohailsa@hotmail.com

\section{Introduction}

Severe acute respiratory syndrome coronavirus 2 (SARS-CoV-2) emerged in late 2019 in Wuhan, China ${ }^{1}$. This disease was declared as global health emergency by WHO on 30th January 2020. ${ }^{2}$ According to the published literature, older patients with underlying illness such as diabetes mellitus, hypertension, and Ischemic heart disease tend to be susceptible to COVID-19 and become severely ill. ${ }^{2}$ Patients with ESRD have abnormal 


\section{COVID-19 \& Hemodialysis}

immune systems because of the uremic state, which is often accompanied by significant comorbidities like cardiovascular disease, diabetes, and cerebrovascular disease. ${ }^{3}$

In this study, we retrospectively collected and analyzed clinical data from all patients undergoing maintenance hemodialysis identified as having laboratory-confirmed COVID-19 distributed in AFIU. The Clinical features of COVID-19 in patients undergoing MHD are presented, and the effectiveness of intervention measures for pandemic control of COVID-19 in MHD centers is reviewed.

\section{Methods}

Data for this prospective, observational study was obtained on telephone. We screened all our hemodialysis dependent patients. Nasopharyngeal swabs were taken for PCR of covid-19. In total, between March 1 and August 15, 2020, 39 of 268 patients undergoing MHD in AFIU were identified as having laboratory confirmed COVID-19 and were enrolled in our study.

\section{Clinical Classification}

Suspected case: A suspected case can be diagnosed with any one of the epidemiological history

a) Travel history or residence in areas where local cases continue to spread within 14 days before the onset.

b) Suspected contact with people who tested positive for COVID-19 or patients with fever or respiratory symptoms within 14 days; and/or respiratory symptoms alone: Cough, Shortness of breath or difficulty breathing; or at least two of these symptoms: fever, chills, repeated shaking Confirmation of cases: with chills, muscle pain, headache, sore throat, new loss of taste or smell. ${ }^{4}$

a. RT- PCR test of throat swab samples positive for COVID-19.

b. HRCT chest positive for COVID-19 findings in PCR negative patients who were either symptomatic or asymptomatic having high risk close contact with confirmed cases.

Contact history/exposure with confirm or suspected cases were further classified into high, medium and low risk according to exposure.

a. High Risk: Within two meters without masks for more than 15 minutes repeatedly or continuous sitting in close rooms for more than one hour even with masks.

b. Medium risk: Within one meter with mask for more than 15 minutes to one hour.

c. Low risk: Contact time less than above two categories with masks on Clinical features were recorded to determine frequency. Throat swabs were taken from symptomatic suspected cases and their contacts for COVID-19 RT-PCR to confirm the cases. PCR was done using BGI kits with Tanbead for auto extraction at Armed Forces Institute of pathology. HRCT was performed in PCR negative symptomatic and asymptomatic individuals who were in close contact with confirmed cases (high risk exposure contacts) using Aquilon 128 slice cannon mache 5.

We divided the degree of severity of COVID-19 (mild / moderate / severe versus critical) according to the New Coronavirus Pneumonia Prevention and Control Program. ${ }^{5}$ Mild cases refer to patients who had mild clinical symptoms without manifestation of viral pneumonia on chest CT scans. Moderate refers to cases who had symptoms such as fever and respiratory tract symptoms, etc., with manifestation of viral pneumonia on radiology.

Severe refers to cases who met any of the following criteria:

a) respiratory rate $>30$ breaths $/ \mathrm{min}$

b) oxygen saturation $<93 \%$ at resting state

c) arterial PO2/oxygen concentration $<300 \mathrm{~mm} \mathrm{Hg}$. Patients with pulmonary lesion progression.

Critical patients refer to patients that met any of the following criteria

a) occurrence of respiratory failure requiring ventilatory support

b) presence of shock

c) other multiorgan failure that requires ICU care. 


\section{Data Collection}

We extracted from the registration system exposure history, demographic data, clinical characteristics, laboratory and radiological findings, and management for all patients with lab-confirmed COVID-19 between March 1 and August 15, 2020. We conducted direct communication with patients or their families as much as possible. Verbal consent obtained. The date of disease onset is defined as the date when the symptoms were observed. We got data by direct communication with patients or their families and attending physician. All laboratory testing was performed according to the clinical situation of the patient. Laboratory assessments consisted of a blood complete picture, liver function test, renal function test and CRP. The presence of a radiologic abnormality was determined on the basis of description in documents; if imaging scans were available, they were reviewed by attending doctor. Treatment measures included antiviral or antibiotic therapy, corticosteroid therapy, vitamin $\mathrm{C}$, vitamin $\mathrm{D}$ and mechanical ventilation. Cardiac injury was determined by the serum levels of cardiac biomarkers, or if new abnormalities were shown via electrocardiography.

Statistical analysis was done using SPSS 22.

\section{Results}

From March 1 to August 15, 2020, 268 patients undergoing Maintenance Hemodialysis (MHD) in AFIU, 39 patients were diagnosed as having laboratory confirmed COVID-19. 15 out of 39 were having mild disease, 12 had moderate and 12 had severe disease. Females (54.5\%) were found to be more affected than males (45.5\%). Mean age of patients was 55.9yrs. Duration of hemodialysis was found to be related to the severity of infection. Greater the duration, more the severity of COVID -19 infection was noticed as shown in Table 1.

Table 1: baseline characteristics of 39 maintenance hemodialysis patients who were diagnosed with SARS COV-2 infection.

\begin{tabular}{|l|l|l|l|}
\hline & MILD (n:15) & MODERATE (n:12) & SEVERE (n:12) \\
\hline Age(mean) & $56.2 \mathrm{yrs}$ & $56 \mathrm{yrs}$ & $62.5 \mathrm{yrs}$ \\
\hline $\begin{array}{l}\text { Duration of HD } \\
\text { (Mean, yrs) }\end{array}$ & $\mathbf{6 . 9}$ & $\mathbf{5 . 1}$ & $\mathbf{1 3}$ \\
\hline Male & $20 \%$ & $100 \%$ & $66.7 \%$ \\
\hline Females & $80 \%$ & 0 & $33-3 \%$ \\
\hline
\end{tabular}

In total $41 \%$ patients were smokers. Only $35.8 \%$ of patients were symptomatic while $64.2 \%$ patients were asymptomatic. Dry cough was the commonest symptom (53.8\%) followed by fever (46.1\%) and abdominal pain (18.1\%) as shown in Table 2.

Data from the laboratory findings showed mean Total leucocyte count and platelets 5.5 and 166 respectively. Lymphocytopenia was noticed to be related to severity of illness. Greater the lymphocytopenia, more was the severity of illness. Of all pulmonary CT scans that were performed, the most common abnormities revealed was pleural effusion (45.4\%) that can be contributed to inadequate hemodialysis followed by ground-glass or patchy opacity $(36.3 \%)$. Consolidation in lungs was relatively rare (18.1\%) as depicted in Table 3.

All patients received antibiotics, antiviral, zinc, vitamin C \& D supplement. Oral azithromycin was given to mild cases (45.5\%). Injectable antibiotics were added in moderate and severe cases (54.5\%) while steroid were given to severe/critical cases only (27.2\%) as per protocol of infectious disease and critical care specialists.

\section{Discussion}

To the best of our knowledge, this is the first multicenter study in Pakistan to focus on patients undergoing MHD who have had COVID-19 .We prospectively collected clinical data of 39 patients undergoing MHD with laboratory confirmed COVID-19, and reviewed intervention required for controlling the spread of the pandemic. Chronic kidney disease is a global public health problem with poor outcomes, and high cost. ${ }^{6,7}$

Kidney disease (CKD) is an independent risk factor for cardiovascular disease (CVD) and infection. ${ }^{8.9}$ Patients are highly susceptible to COVID-19 and there is a high risk of spread of disease in MHD centers. In the current study, 35.8\% of COVID -19 positive patients were symptomatic while rest of the patients were asymptomatic. 
Table 2: Comorbidities and clinical features of 39 maintenance hemodialysis patients.

\begin{tabular}{|c|c|c|c|c|}
\hline & \multicolumn{3}{|c|}{$\begin{array}{c}\text { Severity } \\
\mathrm{n}(\%)\end{array}$} & \multirow[t]{2}{*}{ p value } \\
\hline & Mild & Moderate & Severe & \\
\hline \multicolumn{5}{|c|}{ Frequency of dialysis } \\
\hline $2 / 7$ & $9(60)$ & $12(100)$ & $4(33.3)$ & \multirow[t]{2}{*}{0.003} \\
\hline $3 / 7$ & $6(40)$ & - & $8(66.7)$ & \\
\hline \multicolumn{5}{|l|}{ Fever } \\
\hline Yes & $6(40)$ & - & $12(100)$ & \multirow[t]{2}{*}{$<0.001$} \\
\hline No & $9(60)$ & $12(100)$ & - & \\
\hline \multicolumn{5}{|c|}{ Diabetes Mellitus } \\
\hline Yes & $9(60)$ & $8(66.70)$ & $8(66.70)$ & \multirow[t]{2}{*}{0.915} \\
\hline No & $6(40)$ & $4(33.3)$ & $4(33.3)$ & \\
\hline \multicolumn{5}{|l|}{ Hypertension } \\
\hline Yes & $15(100)$ & $12(100)$ & $8(66.7)$ & \multirow[t]{2}{*}{0.007} \\
\hline No & - & - & $4(33.3)$ & \\
\hline \multicolumn{5}{|c|}{ Ischemic Heart disease } \\
\hline Yes & $6(40)$ & $8(66.7)$ & $12(100)$ & \multirow[t]{2}{*}{0.005} \\
\hline No & $9(60)$ & $4(33.3)$ & - & \\
\hline \multicolumn{5}{|l|}{ Smoking status } \\
\hline Yes & - & $8(66.7)$ & $8(66.7)$ & \multirow[t]{2}{*}{0.001} \\
\hline No & $15(100)$ & $4(33.3)$ & $4(33.3)$ & \\
\hline \multicolumn{5}{|l|}{ Cough } \\
\hline Yes & $9(60)$ & - & $12(100)$ & \multirow[t]{2}{*}{0.001} \\
\hline No & $6(40)$ & $12(100)$ & - & \\
\hline \multicolumn{5}{|l|}{ Dyspnoea } \\
\hline Yes & - & 4 (33.3) & $12(100)$ & \multirow[t]{2}{*}{0.001} \\
\hline No & $15(100)$ & $8(66.7)$ & - & \\
\hline \multicolumn{5}{|l|}{ Vomiting } \\
\hline Yes & - & - & $8(66.7)$ & \multirow[t]{2}{*}{0.001} \\
\hline No & $15(100)$ & $12(100)$ & $4(33.3)$ & \\
\hline \multicolumn{5}{|l|}{ Sorethroat } \\
\hline Yes & - & $8(66.7)$ & $12(100)$ & \multirow[t]{2}{*}{0.001} \\
\hline No & $15(100)$ & $4(33.3)$ & - & \\
\hline Ground glass op & & & & \\
\hline Pleural effusion & $15(100)$ & $4(33.3)$ & - & 0.001 \\
\hline $1+2+3$ & - & $4(33.3)$ & - & \\
\hline $2+3$ & - & $4(33.4)$ & - & \\
\hline $1+3$ & - & - & $12(100)$ & \\
\hline Steroids & & & & \\
\hline Yes & - & $4(33.3)$ & $12(100)$ & 0.001 \\
\hline No & $15(100)$ & $8(66.7)$ & - & \\
\hline Mechanical Ven & & & & \\
\hline Yes & - & - & $8(66.7)$ & 0.001 \\
\hline No & $15(100)$ & $12(100)$ & $4(33.3)$ & \\
\hline Alive & & & & \\
\hline Yes & $15(100)$ & $12(100)$ & $4(33.3)$ & 0.001 \\
\hline No & - & - & $8(66.7)$ & \\
\hline Dead & & & & \\
\hline Yes & - & - & $8(66.7)$ & 0.001 \\
\hline No & $15(100)$ & $12(100)$ & $4(33.3)$ & \\
\hline
\end{tabular}

Therefore, universal screening of patients is necessary to control the pandemic in patients undergoing MHD and should be done at the earliest opportunity. Patients on MHD have impaired immune systems and are at already high risk of infection, including serious complications from infection and increased mortality rates. Infection has become the second most common cause of death in patients with ESRD after CVD. ${ }^{10}$ In addition to bacterial infections, ESRD patients on MHD are also susceptible to viral infections like hepatitis B and C virus, HIV or influenza virus infection. On the other hand, there are additional difficulties in the management of ESRD patients on maintenance hemodialysis who have COVID-19. For example, there is infection control problems in preventing infection spread within the dialysis unit and dealing with the disposal of used dialysate. There were 


\section{COVID-19 \& Hemodialysis}

more women than men in the group of patients infected unlike the study in China in which males were found to be more affected. ${ }^{5}$ We also noticed that patients with cardiovascular disease were more likely to develop severe disease and there is no significant differences in severity of disease in diabetic patients which is in accordance with study of china. ${ }^{5}$ The dialysis access, \& frequency of dialysis had significant effect on the degree of disease severity.

Considering the high risk of spread of disease in a dialysis setup, strict policies regarding entrance screening of temperatures, and the need for all the patients to wear surgical masks, are necessary since symptoms of covid patients who are on MHD were similar to those of the general population. However, these symptoms are difficult to distinguish from uremic symptoms. Only $46.1 \%$ of patients experienced fever and $53.8 \%$ had cough and $64.2 \%$ patients were asymptomatic over the whole clinical course. This finding is consistent with results of study done in Spain and China. ${ }^{5,11}$ Mean age of our patients was 55.9 years versus 63.2 years in the Chinese study while, with high prevalence of hypertension (91\% vs $68.7 \%$ reported in Chinese study) and diabetes (63.6\% vs $22.9 \%$ reported in Chinese study). ${ }^{5}$ Similar findings were reported from study done in Newyork. ${ }^{12}$

The representative findings in the radiological imaging of patients undergoing MHD with COVID-19 were similar to those in the general population, but sometimes it was difficult to distinguish them from lung changes caused by inadequate dialysis. Lymphocytopenia was common in our patients. Previous studies have suggested that the severity of lymphocytopenia shows the severity of SARS-COV-2 infection ${ }^{5,13,14}$ which is also noticed in our study. The uremic state is associated with a wide range of impairments in blood cells predominantly lymphocytes and granulocytes, and lymphocytopenia is common in dialysis patients. So, whether or not lymphocytopenia helps us to identify SARS-CoV-2 in the early phase of infection needs further evaluation. The tissue damage seen in patients with COVID-19 is considered to be caused mainly by a cytokine storm; it is a possibility that abnormalities in the immune system of ESRD patients could predispose patients on MHD to a modified response to SARS-CoV-2 infection.

Four of our medical staff including 2 nurses and 2 ward boys were also infected during this duration of study highlighting that the effective measures to be taken as soon as possible for preventing disease spread. ${ }^{15,16}$ It should be noted that the universal screening of PCR of patients is critically important in helping the identification of asymptomatic COVID-19 patients in dialysis centers, especially during the early phase of the pandemic.

\section{Conclusion:}

Covid -19 patients can be asymptomatic so effective measures for screening should be followed secondly Dialysis centers are at high risk for cross contamination of disease in other patients and medical staff so detection of disease in asymptomatic patients is necessary.

\section{Conflict of Interest: None declared}

\section{References:}

1. Kliger AS, Silberzweig J Mitigating risk of COVID-19 in dialysis facilities. Clin J Am Soc Nephrol 15: 707 - 709, 2020. DOI: https://doi.org/10.2215/CJN.03340320

2. Zeb S, Umar M, Shahid R, Aziz Q, Akram MO, Khurrum M et al., An Overview of Preliminary COVID-19 Cases Admitted in Rawalpindi Institute of Urology \& Transplantation Pakistan. Res \& Rev Health Care Open Acc J 5(2)- 2020. doi: 10.32474/RRHOAJ.2020.05.000210

3. Zhai P, Ding Y, Wu X. The epidemiology, diagnosis and treatment of COVID-19. Int J Antimicrob Agents. 2020;55(5):105955. doi: 10.1016/j.jijantimicag.2020.105955.

4. Covid.gov.pk. 2020 [cited 19 July 2020]. Available from: http://covid.gov.pk/. Clinical Management Guidelines for COVID19 Infections: http://covid.gov.pk/. (Accessed on 19 July 2020).

5. Betjes MG. Immune cell dysfunction and inflammation in end-stage renal disease. Nat Rev Nephrol 9:255 - 265. doi: 10.1038/nrneph.2013.44

6. X.Fei, T. Hui, L. Li, T. Can , T. Jian-Bo, T. Chun, et all,JClinical Characteristics of and Medical Interventions for COVID-19 in Hemodialysis Patients in Wuhan, China. J Am Soc Nephrol 2020, 31 (7) 1387-1397; DOI: https://doi.org/10.1681/ASN.2020030354 


\section{COVID-19 \& Hemodialysis}

7. Hill NR, Fatoba ST, Oke JL, et al. Global Prevalence of Chronic Kidney Disease - A Systematic Review and MetaAnalysis. PLoS One. 2016;11(7):e0158765. Published 2016 Jul 6. https://doi.org/10.1371/journal.pone.0158765

8. Di Napoli A, Pezzotti P, Di Lallo D, et al. Determinants of hospitalization in a cohort of chronic dialysis patients in central Italy. J Nephrol. 2005;18(1):21-29.

9. Imtiaz S, Qureshi R, Hamid A, Salman B, Drohlia MF, et al. Causes of hospital admission of chronic kidney disease patient in a tertiary kidney care hospital. J Clin Nephrol. 2019;3:100-106. DOI: 10.29328/journal.jcn.1001033

10. Hsu HW, Lang CL, Wang MH, Chiang CK, Lu KC-A Review of Chronic Kidney Disease and the Immune System: A Special Form of Immunosenescence. J Gerontol Geriat Res 2014;3:144. doi:10.4172/2167-7182.1000144

11. G.Marian ,A. Luis Alberto, M. Nicolá, M.Alejandra,R. Á ngela Gonza,et all, COVID-19: clinical course and outcomes of 36 hemodialysis patients in Spain. Kidney Int 2020;98:27 - 34. doi.org/10.1016/j.kint.2020.04.031

12. M. Valeri Anthony. Y. Robbins-Juarez Shelief, S.Jacob,et all, Presentation and Outcomes of Patients with ESKD and COVID19. J Am Soc Nephol 2020;31 (7):1409-1415. DOI: https://doi.org/10.1681/ASN.2020040470

13. E.Bingwen,C.Vanessa,S.Stephrene,H.Gek,et all, Hematologic parameters in patients with COVID-19 infection. Am J Hematol. 2020 Jun;95(6):E131-E134. doi: 10.1002/ajh.25774.

14. Chng WJ, Lai HC, Earnest A, Kuperan P. Haematological parameters in severe acute respiratory syndrome. Clin Lab Haematol. 2005;27:15 - 20. doi: 10.1111/j.1365-2257.2004.00652.x.

15. W Jun, L Jushuang, Z Geli, Z Yanxia, B Zhimin. Clinical Features of Maintenance Hemodialysis Patients with 2019 Novel Coronavirus-Infected Pneumonia in Wuhan, China. Clin J Am Soc Nephrol. 2020;15(8): 1139-1145; DOI: https://doi.org/10.2215/CJN.04160320.

16. Guan WJ, Ni ZY, Hu Y, Liang WH, Ou CQ, He JX et al. China Medical Treatment Expert Group for Covid-19. Clinical characteristics of coronavirus disease 2019 in China. N Engl J Med 2020;382(18):1708 - 1720 doi: 10.1056/NEJMoa2002032. 